\title{
Risk factors of hospitalisation after outpatient shoulder surgery
}

\author{
Ousmane Bal ${ }^{1}$, Gustave Talla ${ }^{1}$, Sofiane Boulares ${ }^{2}$, Laurence Seidel ${ }^{3}$, Jean Boogaerts ${ }^{1}$ \\ Departments of ${ }^{1}$ Anaesthesia and ${ }^{2}$ Orthopedics, University Hospital Centre of Charleroi, \\ and ${ }^{3}$ Department of Biostatistics, University of Liege, Belgium
}

Introduction: Shoulder arthroscopy is one of the most commonly performed procedures in orthopedics for a multitude of surgical indications and is now routinely performed as an outpatient basis $(1,2)$. The aim of this observational study is to evaluate the risk factors leading to hospitalisation.

Methods: We included consecutively all patients, undergoing shoulder arthroscopy under general anaesthesia combined with an interscalene nerve block in a routine ambulatory setting after premedication with alprazolam. General anaesthesia was induced with propofol, sufentanil and rocuronium for endotracheal intubation. Sevoflurane was associated with reinjection of opioids and myorelaxant as required. At the end of surgery, patients received paracetamol and ketorolac. After surgery, they were placed in postanaesthesia care unit (PACU) before leaving hospital or being hospitalised. The following variables were registered: demography, hours of induction, length of surgery, anaesthetic or analgesic consumption during and after surgery, EVA pain scores, PONV, time to refeeding, length of stay in PACU and side effects. Patient satisfaction was rated on a five-point Likert scale. Mean SD or percentages. Student's t, Chi2 tests and logistic regression for statistical analysis.

Results: A total of 151 patients were included in the study. Among them, 27 (18\%) were hospitalised. The factors related to hospitalisation are gender, BMI, ASA scores > II, time of surgery after 10 am, use of myorelaxant, duration of regional block and PACU stay. No significant difference in the other variables. Results are presented in following tables.

Table 1. Demography $(\mathrm{N}=151)$

\begin{tabular}{lcccc}
\hline Variables & out patient & hospitalisation & OR [IC 95\%] & P \\
\hline Gender & & & & 0.009 \\
$\quad$ female $(\mathrm{n})$ & $68(91 \%)$ & $7(9 \%)$ & 1 & \\
$\quad$ male $(\mathrm{n})$ & $56(74 \%)$ & $20(26 \%)$ & $3.47[1.37-8.80]$ & 0.15 \\
Age (yrs) & $51.3 \pm 11.6$ & $54.8 \pm 10.9$ & $1.03[0.99-1.07]$ & 0.04 \\
BMI (kg/m $)$ & $27.4 \pm 4.7$ & $29.8 \pm 7.0$ & $1.08[1.004-1.17]$ & $<0.001$ \\
ASA class & & & 1 & \\
I (n) & $47(96 \%)$ & $2(4 \%)$ & $6.03[1.34-27.1]$ & \\
II (n) & $74(80 \%)$ & $19(20 \%)$ & $47.00[6.48-341]$ & \\
III (n) & $3(33 \%)$ & $6(67 \%)$ & &
\end{tabular}

$\mathrm{BMI}=$ Body Mass Index; ASA = American Society of Anesthesiologists

Table 2. Peroperative data $(\mathrm{N}=151)$

\begin{tabular}{|c|c|c|c|c|}
\hline Variables & out patient & hospitalisation & OR [IC 95\%] & $P$ \\
\hline Alprazolam (mg) & $0.50 \pm 0.97$ & $0.93 \pm 1,21$ & $1.43[0.99-2.06]$ & 0.056 \\
\hline Induction time & & & & 0.002 \\
\hline$<10$ am $(\mathrm{n})$ & $68(96 \%)$ & $3(4 \%)$ & 1 & \\
\hline $10-12$ am (n) & $39(71 \%)$ & $16(29 \%)$ & 9.30 [2.55-3.9] & \\
\hline$>12$ am (n) & $17(68 \%)$ & $8(32 \%)$ & $10.70[2.55-44.5]$ & \\
\hline Chirocaïne (mg) & $83.35 \pm 33.47$ & $96.11 \pm 44.17$ & 1.01 [0.99-1.02] & 0.10 \\
\hline Propofol (mg) & $164.24 \pm 34.72$ & $175.56 \pm 45.26$ & 1.01 [0.99-1.02] & 0.15 \\
\hline Sufentanil ( $\mu g$ ) & $10.65 \pm 3.14$ & $10.17 \pm 3.11$ & 0.95 [0.83-1.10] & 0.48 \\
\hline Rocuronium (mg) & $18.39 \pm 14.05$ & $30.00 \pm 9.21$ & 1.08 [1.03-1.13] & $<0.001$ \\
\hline Surgery duration (min) & $63.50 \pm 21.57$ & $69.31 \pm 24.32$ & 1.01 [0.99-1.03] & 0.23 \\
\hline
\end{tabular}

Table 3. Postoperative data $(\mathrm{N}=151)$

\begin{tabular}{|c|c|c|c|c|}
\hline Variables & out patient & hospitalisation & OR [IC 95\%] & $P$ \\
\hline PACU duration (min) & $47.83 \pm 20.15$ & $64.44 \pm 23.47$ & 1.03 [1.01-1.05] & 0.001 \\
\hline Bloc duration (h) & $18.74 \pm 7.95$ & $22.77 \pm 10.12$ & 1.06 [1.01-1.11] & 0.03 \\
\hline Refeeding (min) & $98.27 \pm 51.30$ & $110.00 \pm 41.69$ & 1.00 [0.99-1.01] & 0.27 \\
\hline AUC-VAS $\left(\mathrm{cm}^{2}\right)$ & $16.92 \pm 23.67$ & $16.83 \pm 32.13$ & $1.00[0.98-1.02]$ & 0.99 \\
\hline $\operatorname{PONV}(n)$ & $14(87.7 \%)$ & $2(12.5 \%)$ & $0.65[0.14-3.05]$ & 0.58 \\
\hline Satisfaction (1-5) & $4.56 \pm 0.89$ & $4.46 \pm 1.14$ & 0.90 [0.59-1.38] & 0.62 \\
\hline
\end{tabular}

PACU = Post Anaesthesia Care Unit; AUC = Area Under the Curve; VAS = Visual Analogue Scale; PONV = Postoperative Nausea and Vomiting;

Discussion: Results of this study show that a more deliberate use of myorelaxant is associated with a larger percentage of hospitalisation after outpatient shoulder surgery. It should be mentioned that no myorelaxant antagonist has been administered at the end of anaesthesia. Likewise it appears that hospitalised patients suffered higher ASA scores and stayed longer in PACU.

References : 1. Mather et al. J Bone Joint Surg 2013;95:1993-2000. ; 2, Warrender et al. Am J Sports Med published online October 11.2016. 